\title{
HAl Surveillance Enhancement within EpiCenter by Utilization of Triage Notes
}

\author{
Pinar Erdogdu*, Stella Tsai and Teresa Hamby \\ New Jersey Department of Health, Trenton, NJ, USA
}

\section{Objective}

Evaluate the usage of triage note data from EpiCenter, a syndromic surveillance system utilized by New Jersey Department of Health (NJDOH), to enhance Healthcare-Associated Infections (HAIs) surveillance for infections following a surgical procedure.

\section{Introduction}

In New Jersey, Health Monitoring Systems Inc.'s (HMS) EpiCenter collects chief complaint data for syndromic surveillance from 79 of 80 emergency departments (ED). Using keyword algorithms, these visits are classified into syndrome categories for monitoring unusual health events.

HAIs are infections that patients acquire while they are receiving treatment for a health condition in a health care setting. Following the 2014 Ebola outbreak in West Africa, the New Jersey Department of Health (NJDOH) Communicable Disease Service (CDS) started recruiting EDs to include triage note data in addition to chief complaint data to enhance surveillance capability for Ebola and other HAIs. Research by the University of North Carolina suggests triage note data improve the ability to detect illness of interest by fivefold ${ }^{1}$. Currently, there are three NJ EDs with triage note data in EpiCenter along with ICD 10 codes which can be used for comparison.

This pilot study will assess whether infections following a surgical procedure can be captured in triage note data along with ICD codes. Also, this evaluation will determine if triage note data can be used to create HAI custom classifications for syndromic surveillance. These classifications can potentially be used by surveillance and/or preparedness personnel and local health departments, as well as hospitals, to better prepare for detecting and preventing HAIs that are a significant cause of morbidity and mortality in the U.S. ${ }^{2}$

\section{Methods}

Three NJ facilities with triage notes information sending to EpiCenter were included in this study. ED visits occurred from $10 / 23 / 2015$ to $10 / 29 / 2015$ and from $2 / 2 / 2016$ to $2 / 10 / 2016$ in these facilities with available ICD 10 codes information in EpiCenter were evaluated.

This analysis focused on sepsis and post-surgery infections related ICD 10 codes: A400, A401, A402, A403, A408, A409, A410, A411, A412, A414, A4150, A4151, A4152, A4158, A418, A419, R571, R578, R579, T811, T81.4³. The keywords tested in triage notes are abdominal pain, redness, fev, fver, pyrexia, temp, elev temp, elevated temp, temp elev, hi temp, high temp, temp hi, temp10, temp 10, feeling hot, feels hot, feel hot, fuo, febr, cloudy fluid, cfluid, drainage, abscess, wound, tenderness, swelling, erythema, red, pain, post surgery, fever.

The sensitivity, specificity and positive predictive value (PPV) of selected keywords applied in the triage notes were evaluated by comparing to patient's ICD 10 codes.

\section{Results}

There were $2757 \mathrm{ED}$ visits with triage notes and ICD 10 codes from $10 / 23 / 2015$ to $10 / 29 / 2015$ and from $2 / 2 / 2016$ to $2 / 10 / 2016$. During these time frames, one ED visit matched with both selected keywords and ICD codes, five matched with ICD 10 codes only, 59 matched with keywords only, and 2692 did not match with either keywords or ICD 10 codes. In Table 1 , it indicates that selected keywords have a high specificity $(97.9 \%)$ but with a relatively low sensitivity $(16.7 \%)$ and PPV (1.7\%).

\section{Conclusions}

Selected keywords and ICD 10 codes from facilities sending triage notes were used to evaluate the surveillance system on identifying infections following a surgical procedure through analysis of ED triage note field. We also reviewed all NJ ED data during the same study period for other facilities not sending triage notes. It indicated that several key ICD codes, e.g. ICD code T81.4, infections following a surgical procedure, have been included in many facilities. This analysis will be repeated as more EDs participate in EpiCenter with triage notes and other data fields to refine the keywords and to improve the sensitivity and PPV.

\begin{tabular}{|c|c|c|c|c|}
\hline \multirow{2}{*}{\multicolumn{2}{|c|}{$\begin{array}{l}\text { Sensitivity: } \mathbf{1 6 . 7} \% \\
\text { Specificity: } \mathbf{9 7 . 9} \% \\
\text { PPV: } 1.7 \%\end{array}$}} & \multicolumn{2}{|c|}{ ICD 10} & \multirow[b]{2}{*}{ Total } \\
\hline & & Matched & Not Matched & \\
\hline \multirow[t]{3}{*}{ Keywords } & Matched & 1 & 59 & 60 \\
\hline & Not Matched & 5 & 2692 & 2697 \\
\hline & Total & 6 & 2751 & 2757 \\
\hline
\end{tabular}

Table 1: Sensitivity, specificity and PPV calculations of selected keywords applied in triage notes based on the ICD 10 codes related to infections following a surgical procedure.

\section{Keywords}

Healthcare-Associated Infections; Surgical Site Infections; EpiCenter; New Jersey

\section{Acknowledgments}

Elizabeth Kostial, Kristen Weiss and Jason Liggett (HMS).

\section{References}

1. Travers DA, Barnett C, Ising A, Waller A. Timeliness of emergency department diagnoses for syndromic surveillance. Proceedings of the American Medical Informatics Association; 2006 :769-773.

2. Healthy People 2020. Washington, DC: U.S. Department of Health and Human Services, Office of Disease Prevention and Health Promotion. Date URL was accessed July 12 2016. Available from: https://www.healthypeople.gov/2020/topics-objectives/topic/ healthcare-associated-infections

3. Health Quality Measures of New Zealand. Excess Length of Stay Associated with Postoperative Sepsis. Date URL was accessed July 12 2016. Available from: http://www.hqmnz.org.nz/library/Excess Length_of_Stay_Associated_with_Postoperative_Sepsis

\footnotetext{
*Pinar Erdogdu

E-mail: pinar.erdogdu@doh.nj.gov
} 